\title{
OPEN Quercetin-treated rat sperm enables refrigerated transport with motility and fertility for five days
}

\author{
Katsuma Yamaga ${ }^{1}$, Satohiro Nakao ${ }^{1}$, Nobuyuki Mikoda ${ }^{2}$, Hidetaka Yoshimoto ${ }^{1}$, \\ Ena Nakatsukasa ${ }^{3}$, Naomi Nakagata ${ }^{2} \&$ Toru Takeo $^{1 \bowtie}$
}

Shipment of laboratory rats between animal facilities is frequently performed using special containers. However, the shipment of live animals is associated with potential risks of infectious diseases, escape and death during shipment and animal welfare issues. The transport of cold-stored sperm avoids such risks; however, there have been no reports on cold storage of rat sperm. We previously reported that dimethyl sulfoxide (DMSO) and quercetin maintained the motility and fertilising abilities of coldstored mouse sperm stored for 10 days. The present study investigated the efficacy of DMSO and quercetin in the cold storage of rat sperm. Quercetin maintained motility and fertility of cold-stored rat sperm stored for 5 days. After in vitro fertilisation using cold-stored sperm, pronuclear and two-cell embryos developed normally to pups following embryo transfer. Therefore, we demonstrated that live pups could be obtained from sperm transported using the cold-storage system. We conclude that cold storage of rat sperm may provide an efficient system for transporting rat resources as an alternative to shipping live animals.

Genetically engineered rats are frequently transported between animal facilities in special containers. However, the shipment of live animals is associated with potential risks, such as spreading infectious diseases, escape and death of animals and animal welfare issues. On the other hand, the transport of cryopreserved sperm and embryos could be a useful means to avoid such risks. The cryopreservation of sperm and embryos requires ultra-low temperatures using special containers (dry shippers) and proficient skills to handle the cryopreserved samples $^{1,2}$. Furthermore, the use of freeze-dried sperm in the production of pups via intracytoplasmic sperm injection is associated with technical barriers ${ }^{3}$. Simple and user-friendly techniques are required to transport genetically engineered rats to overcome these issues.

The transport of cold-stored sperm is potentially useful for the shipment of mutant rats. However, the motility of cold-stored rat sperm rapidly decreases and there may be damage to the acrosomal membrane and DNA in Tyrode lactate-HEPES, Ham's F10 medium plus raffinose or trehalose or fructose, tris (hydroxymethyl) aminomethane (Tris)-citrate, Krebs-Ringer bicarbonate, lactose-egg yolk and phosphate-buffered saline at $4{ }^{\circ} \mathrm{C}$ within $24 \mathrm{~h}^{4-6}$. We previously developed a cold-storage system for mouse sperm ${ }^{7}$. Mouse sperm stored in coldstorage medium of Lifor supplemented with dimethyl sulfoxide (DMSO) and quercetin at $4{ }^{\circ} \mathrm{C}$ maintained motility and fertility for 10 days $^{8}$. The protective effects of DMSO and quercetin may be applicable to rat sperm; however, there have been no previous reports about the cold-storage of rat sperm or in vitro fertilisation (IVF) using cold-stored rat sperm.

The present study examined the efficacy of cold-storage systems for rat sperm. We first examined the effects of quercetin and DMSO on the motility and fertilisation ability of cold-stored sperm. We then examined the fertilisation capacity of sperm stored in cold-storage medium containing DMSO and quercetin for different storage periods. Next, we examined the developmental ability of embryos obtained by IVF using sperm cold-stored

\footnotetext{
${ }^{1}$ Division of Reproductive Engineering, Center for Animal Resources and Development (CARD), Institute of Resource Development and Analysis, Kumamoto University, 2-2-1 Honjo, Chuo-ku, Kumamoto 860-0811, Japan. ${ }^{2}$ Division of Reproductive Biotechnology and Innovation, Center for Animal Resources and Development (CARD), Institute of Resource Development and Analysis, Kumamoto University, 2-2-1 Honjo, Chuo-ku, Kumamoto 860-0811, Japan. ${ }^{3}$ Department of Animal Model Development, Brain Research Institute, Niigata University, 1-757 Asahimachidori, Chuo-ku, Niigata 951-8585, Japan. ${ }^{\circledR}$ email: takeo@kumamoto-u.ac.jp
} 

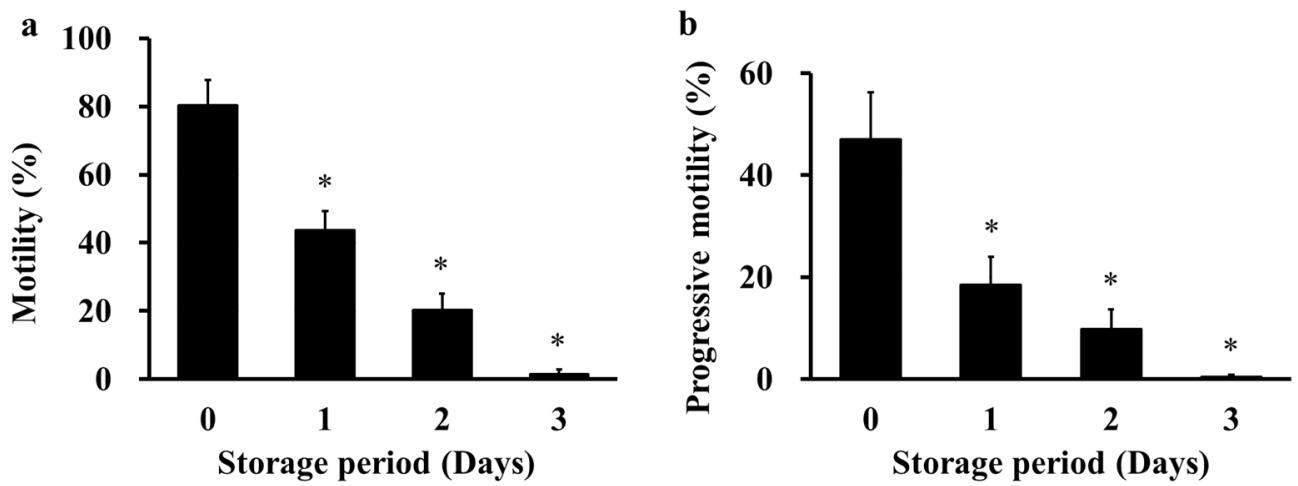

Figure 1. Effect of storage period on motility parameters of cold-stored sperm. Cauda epididymides were cold stored in Lifor at $4{ }^{\circ} \mathrm{C}$ for $0-3$ days. After cold storage, sperm were cultured in mHTF for $2 \mathrm{~h}$ and motility was analysed using an IVOS sperm analyser. Motility was measured as the percentage of sperm moving at a speed of $\geq 5 \mu \mathrm{m} / \mathrm{s}$ (a). Progressive motility was measured as the percentage of sperm moving at $\geq 50 \mu \mathrm{m} / \mathrm{s}$ with a progressiveness of $>50 \%(\mathbf{b})$. Results are expressed as mean \pm SD $(n=3-4$ male rats/group, 14 male rats were used). ${ }^{\star} P<0.05$ compared with day 0 .

with DMSO and quercetin. Finally, we studied the effects of transportation on cold-stored cauda epididymides as well as the fertilisation and developmental abilities of cold-transported rat sperm.

\section{Results}

DMSO and quercetin improved the motility of cold-stored rat sperm. Sperm motility was analysed in sperm cold-stored for 3 days in a cold-storage solution containing various concentrations of DMSO and quercetin to determine their optimal concentrations. We first confirmed how long cold-stored rat sperm could be stored in the cold-storage medium of Lifor and maintain motility. The motility and progressive motility of cold-stored rat sperm stored in Lifor decreased after 1 day (Fig. 1a,b). However, the motility of the cold-stored sperm did not change when stored in Lifor containing $50-300 \mu \mathrm{g} / \mathrm{mL}$ quercetin in 5\% DMSO (Fig. 2a,b). Concentrations of $100-300 \mu \mathrm{g} / \mathrm{mL}$ quercetin in 10\% DMSO increased the percentage motility (Fig. 2c). The progressive motility of the cold-stored sperm did not change with the addition of $100-300 \mu \mathrm{g} / \mathrm{mL}$ quercetin in $10 \%$ DMSO (Fig. 2d). Sperm stored in Lifor containing 150-300 $\mu \mathrm{g} / \mathrm{mL}$ quercetin in 15\% DMSO showed increased motility (Fig. 2e). Furthermore, the progressive motility increased with 200 and $300 \mu \mathrm{g} / \mathrm{mL}$ quercetin in $15 \%$ DMSO (Fig. 2f). The highest rate of motility was observed with Lifor containing $200 \mu \mathrm{g} / \mathrm{mL}$ quercetin in $15 \%$ DMSO.

Cold-storage of rat sperm in $15 \%$ DMSO and $200 \mu \mathrm{g} / \mathrm{mL}$ quercetin improved the fertilisation rate. IVF was performed using sperm cold-stored for 3 days with Lifor containing 15\% DMSO and different concentrations of quercetin to examine the effect of DMSO and quercetin on the fertilising ability of coldstored sperm. Sperm stored with Lifor containing 200 and $300 \mu \mathrm{g} / \mathrm{mL}$ quercetin showed higher rates of total and monospermic fertilisation than sperm stored with Lifor containing DMSO only or $150 \mu \mathrm{g} / \mathrm{mL}$ quercetin (Fig. 3a,b). Rate of polyspermic fertilisation was increased at $300 \mu \mathrm{g} / \mathrm{mL}$ quercetin in $15 \%$ DMSO (Fig. 3c). Therefore, $200 \mu \mathrm{g} / \mathrm{mL}$ quercetin in $15 \%$ DMSO was used for the following experiments based on the results of motility and fertility analysis.

Cold-stored sperm maintained fertilising ability for up to $\mathbf{5}$ days. IVF was performed using sperm cold-stored for 0-6 days to examine the effect of storage period on fertilisation rate. Fertilisation rates gradually decreased after 1 day, but fertilised oocytes were obtained from cold-stored sperm for up to 5 days (Fig. 4a). The monospermic fertilisation rate of fresh and cold-stored sperm was stable for up to 2 days and decreased after 3 days (Fig. 4b). Rates of polyspermic fertilisation of cold-stored sperm were lower than that of fresh sperm. (Fig. 4c).

Motility and progressive motility decreased after 2 days (Fig. 5a,b). Furthermore, the average path velocity (VAP, which shows the average velocity of motile sperm) decreased after 3 days (Fig. 5c). VSL decreased for 4-5 days (Fig. 5d). BCF increased for 4 days (Fig. 5f). ALH and STR were not changed (Fig. 5e,g).

Embryos derived from cold-stored rat sperm developed into live pups. Embryo transfer was performed to evaluate the developmental ability of embryos produced by IVF using fresh and cold-stored sperm (after a cold-storage period of 3 days). Embryos derived from cold-stored sperm developed normally into live pups (Table 1).

Embryos derived from cold-transported sperm developed into live pups. IVF and embryo transfer using cold-transported EGFP-labelled rat sperm were performed to confirm whether sperm retained normal fertilisation and developmental abilities after cold-storage transport from Niigata University to Kumamoto Uni- 
a

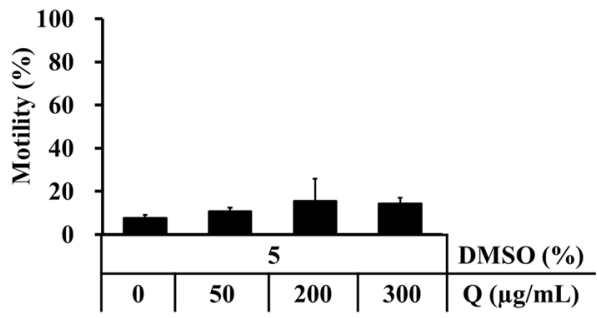

c

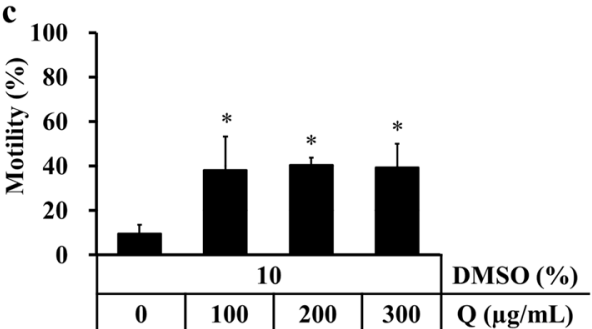

e

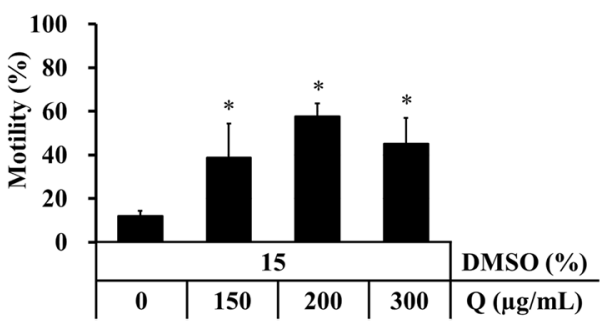

b

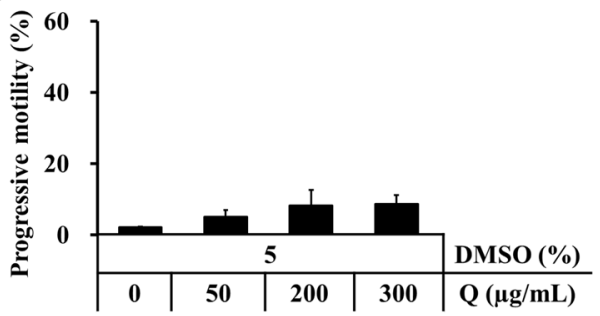

d

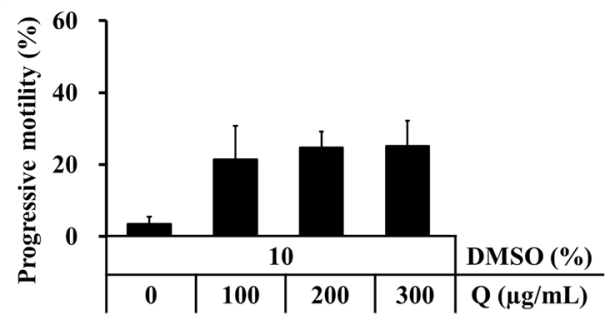

f

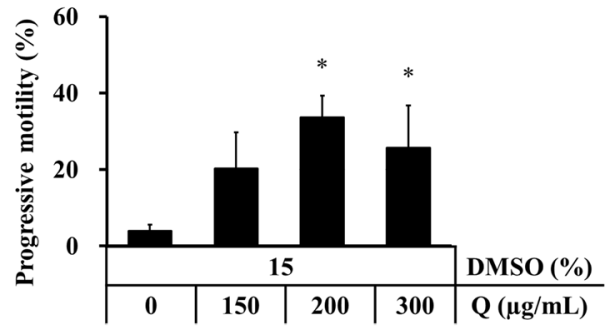

Figure 2. Effect of different concentrations of DMSO and quercetin (Q) on motility of cold-stored sperm. Cauda epididymides were cold-stored in Lifor with various concentrations of DMSO (5\%, 10\% and 15\%) and quercetin $(0,50,100,150,200$ and $300 \mu \mathrm{g} / \mathrm{mL})$ at $4{ }^{\circ} \mathrm{C}$ for 3 days. After cold-storage, sperm were cultured in mHTF for $2 \mathrm{~h}$ and motility was analysed using an IVOS sperm analyser. Motility was measured as the percentage of sperm moving at a speed of $\geq 5 \mu \mathrm{m} / \mathrm{s}(\mathbf{a}, \mathbf{c}, \mathbf{e})$. Progressive motility was measured as the percentage of sperm moving at $\geq 50 \mu \mathrm{m} / \mathrm{s}$ and progressiveness of $>50 \%(\mathbf{b}, \mathbf{d}, \mathbf{f})$. Results are expressed as mean \pm SD $(n=3-5$ male rats/group, 45 male rats were used). ${ }^{\star} P<0.05$ compared with $0 \mu \mathrm{g} / \mathrm{mL}$ quercetin in each experiment.

versity. Fertilised oocytes were obtained by IVF and the embryos developed normally into live pups (Table 2, Fig. 6).

\section{Discussion}

The addition of DMSO and quercetin to cold-storage medium prolonged the motility and fertility of cold-stored rat sperm. Cold-stored rat sperm maintained its fertilising ability for 5 days in vitro. Embryos produced by IVF using the cold-stored rat sperm developed normally to pups following embryo transfer. Furthermore, coldtransported sperm maintained fertility and developmental abilities. This cold-storage technique can be used for the simple and efficient transport of sperm from genetically modified rats.

The present study demonstrated the successful transfer of sperm to a different animal facility and the production of live pups via IVF and embryo transfer from cold-transported rat sperm stored for $64 \mathrm{~h}$. The storage period of cold-stored rat sperm was limited to 5 days, which would be applicable to the domestic or international shipment of genetically modified rats. We recommend shipment on a Monday, with IVF and embryo transfer complete by Friday.

The composition of the storage medium is critical to the yield of embryos after cold storage of rat sperm. Cold-storage medium containing $10 \%$ DMSO and $200 \mu \mathrm{g} / \mathrm{mL}$ quercetin maintained the fertility of rat sperm for 5 days (Fig. 3). We previously reported that cold-storage of mouse sperm in medium containing $10 \%$ DMSO and $100 \mu \mathrm{g} / \mathrm{mL}$ quercetin maintained fertility for 10 days $^{8}$. The protective effects of DMSO and quercetin were previously shown to include alleviation of oxidative stress in rooster and rabbit sperm stored at $4{ }^{\circ} \mathrm{C}$ and $5^{\circ} \mathrm{C}^{9,10}$. In mouse sperm, quercetin was localised in the mitochondria and maintained mitochondrial activity during cold preservation $^{8}$. We assumed that the protective effects of quercetin would be similar in cold-stored rat sperm by reducing oxidative stress and maintaining mitochondrial activity.

DMSO showed a protective effect in cold-stored mouse sperm, whereas it showed no effect in cold-stored rat sperm. DMSO is a polar non-protic solvent that can react with and remove hydroxyl radicals ${ }^{11}$. Excessive production of hydroxyl radicals (a reactive oxygen species) can damage cell membranes and mitochondria, leading to 
a
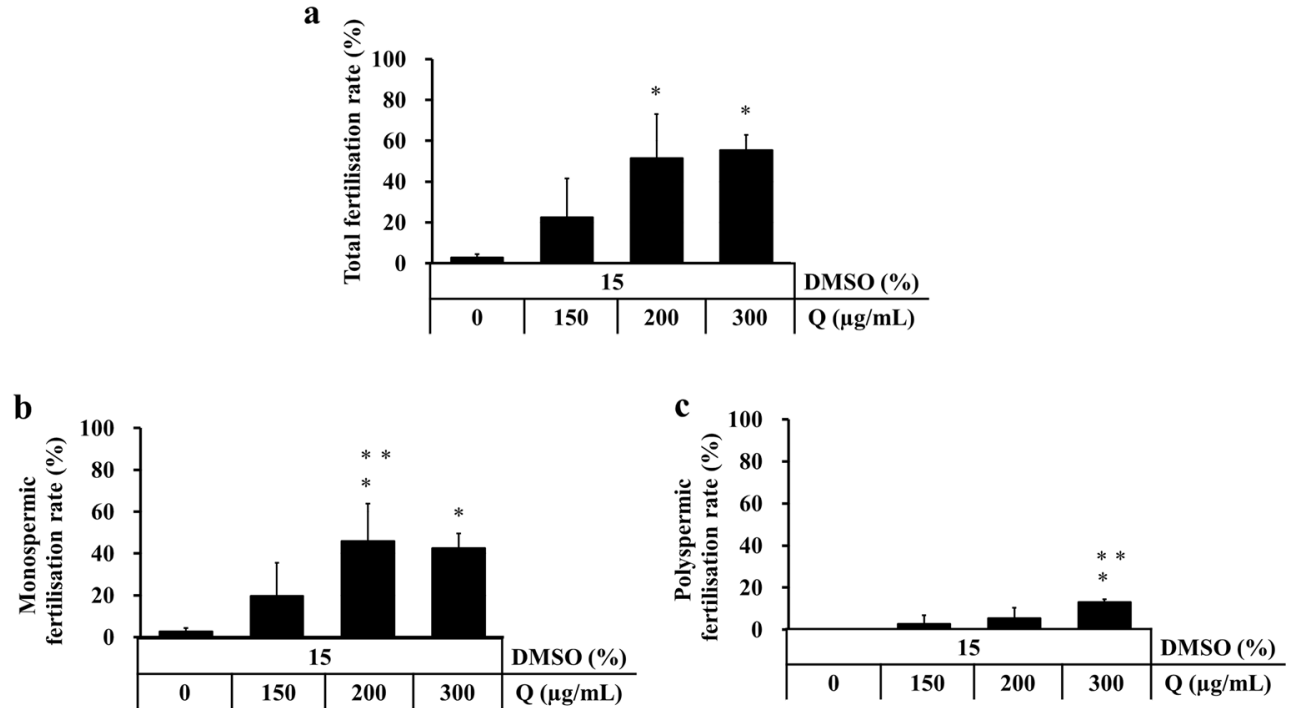

Figure 3. Effect of different concentrations of DMSO and quercetin (Q) on fertility of cold-stored sperm. Cauda epididymides were cold-stored in Lifor with $15 \%$ DMSO and various concentrations of quercetin $(0,150$, 200 and $300 \mu \mathrm{g} / \mathrm{mL}$ ) at $4{ }^{\circ} \mathrm{C}$ for 3 days. After cold-storage, sperm were cultured in $\mathrm{mHTF}$ and cumulus-oocytes complexes collected from a female rat were introduced into the sperm suspension. The total fertilisation rate was calculated as the number of fertilised oocytes divided by the total number of oocytes and multiplied by 100 (a). The monospermic fertilisation rate was calculated as the number of monospermic oocytes (two pronuclei and sperm tail or sperm tail in the cytoplasm) divided by the total number of oocytes and multiplied by 100 (b). The polyspermic fertilisation rate was calculated as the number of polyspermic oocytes (more than three pronuclei or two sperm tails in the cytoplasm) divided by the total number of oocytes and multiplied by 100 (c). Results are expressed as mean \pm SD $\left(n=3-7\right.$ male rats/group, 20 female rats and 20 male rats were used). ${ }^{\star} P<0.05$ compared with $0 \mu \mathrm{g} / \mathrm{mL}$ quercetin. ${ }^{\star *} \mathrm{P}<0.05$ compared with $150 \mu \mathrm{g} / \mathrm{mL}$ quercetin.
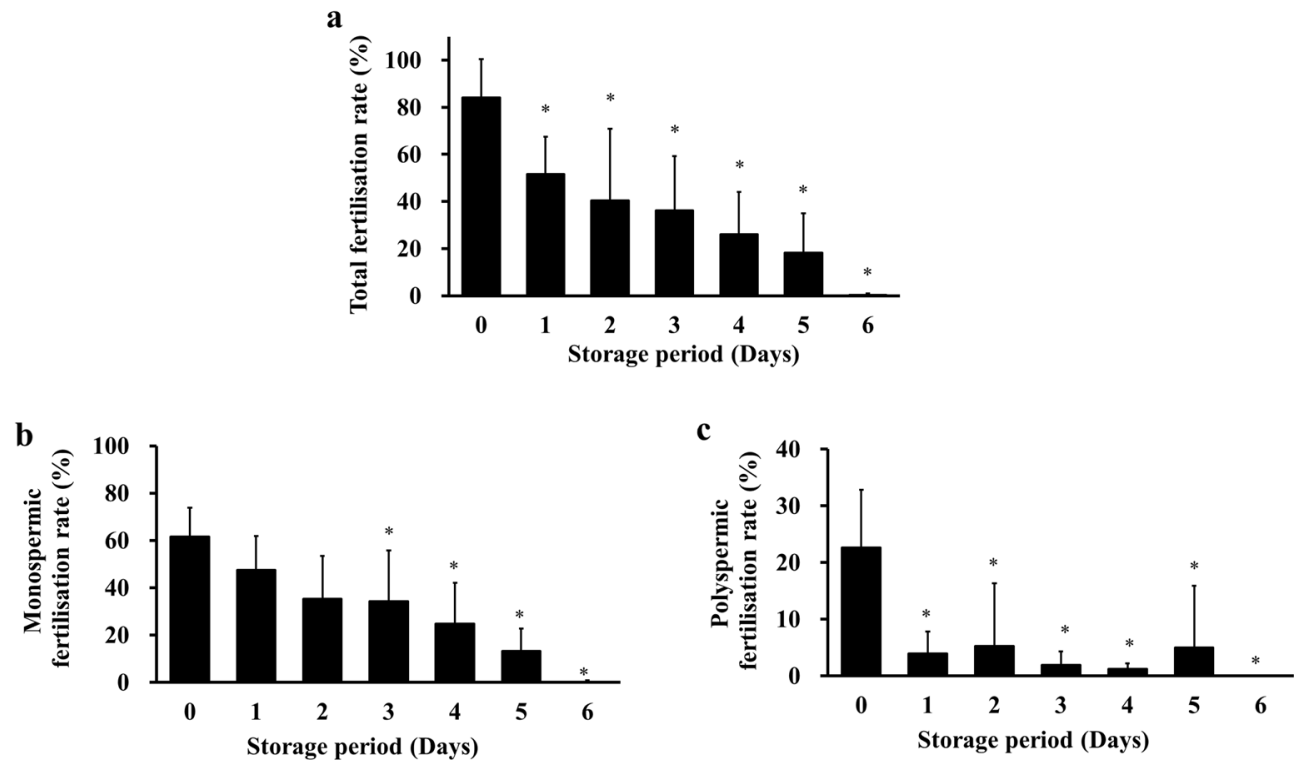

Figure 4. Effect of storage period on the fertilisation ability of cold-stored sperm. Cauda epididymides were cold-stored in Lifor containing 15\% DMSO and $200 \mu \mathrm{g} / \mathrm{mL}$ quercetin at $4{ }^{\circ} \mathrm{C}$ for $0-6$ days. After cold-storage, sperm were cultured in mHTF and cumulus-oocytes complexes collected from a female rat were introduced into the sperm suspension. The total fertilisation rate was calculated as the number of fertilised oocytes divided by the total number of oocytes and multiplied by 100 (a). The monospermic fertilisation rate was calculated as the number of monospermic oocytes (two pronuclei and a sperm tail or a sperm tail in the cytoplasm) divided by the total number of oocytes and multiplied by 100 (b). The polyspermic fertilisation rate was calculated as the number of polyspermic oocytes (more than three pronuclei and more than two sperm tails or more than two sperm tails in the cytoplasm) divided by the total number of oocytes and multiplied by 100 (c). Results are expressed as mean $\pm S D\left(n=4-8\right.$ male rats/group, 46 female rats and 46 male rats were used). ${ }^{\star} P<0.05$ compared with day 0 . 
$\mathbf{a}$

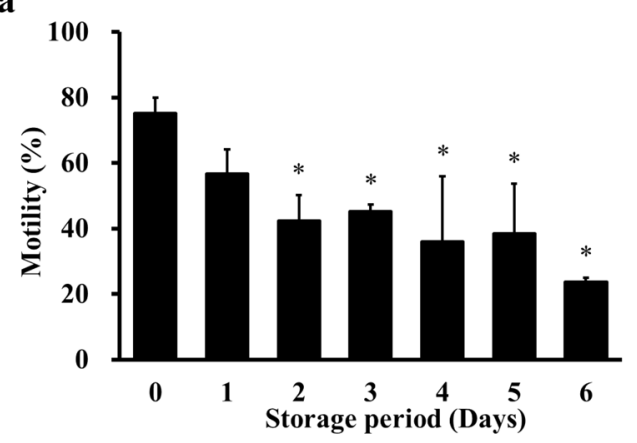

b

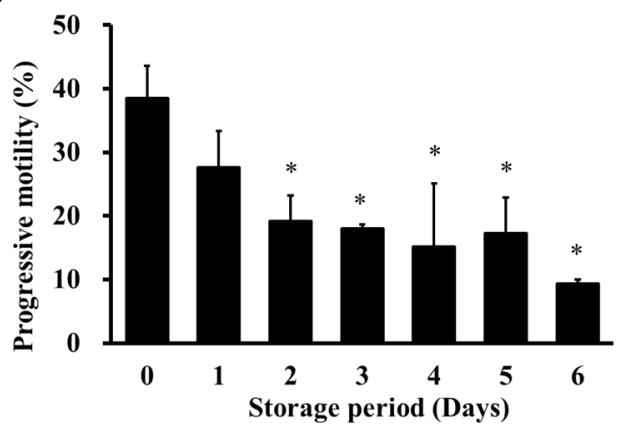

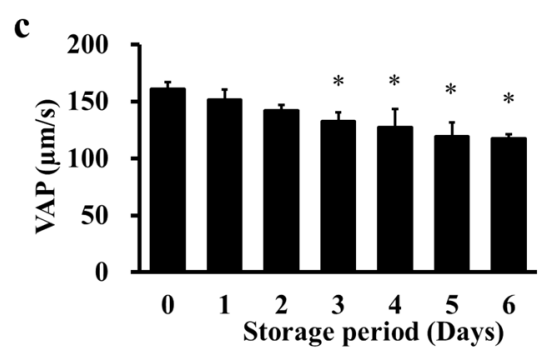

d
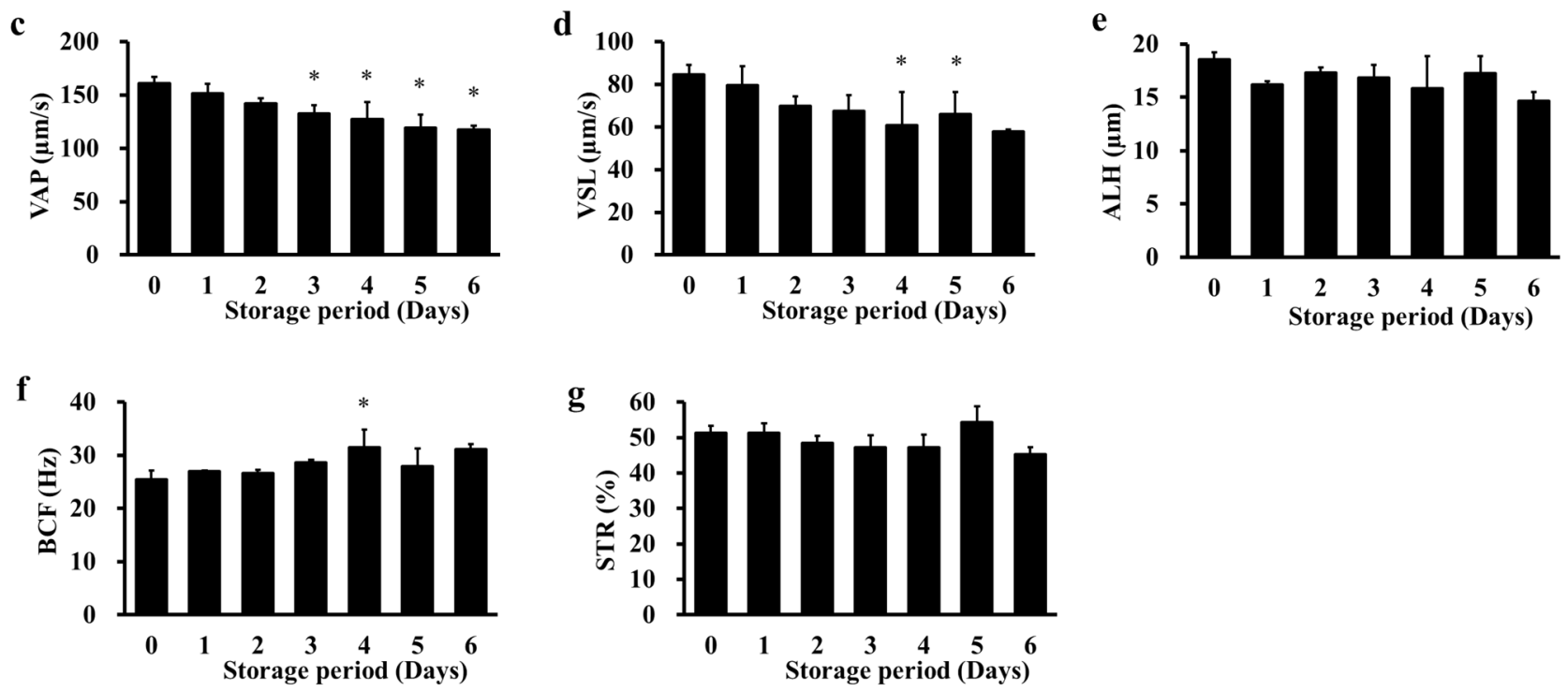

Figure 5. Effect of storage period on the motility of cold-stored sperm. Cauda epididymides were cold-stored in Lifor containing DMSO (15\%) and quercetin $(200 \mu \mathrm{g} / \mathrm{mL})$ at $4{ }^{\circ} \mathrm{C}$ for $0-6$ days. After cold-storage, sperm were cultured in mHTF for $6 \mathrm{~h}$ and the motility was analysed using an IVOS sperm analyser. The following motility parameters were measured: motility (a) and progressive motility (b), VAP (c), VSL (d), ALH (e), BCF (f) and STR (g). Results are expressed as mean \pm SD ( $\mathrm{n}=3-5$ male rats/group, 26 male rats were used). ${ }^{\star} P<0.05$ compared with day 0 .

\begin{tabular}{|l|l|l|l|}
\hline Storage period (day) & No. of recipients & No. of transferred fertilised oocytes & No. of pups (\%) \\
\hline 0 & 3 & 60 & $20(33.3 \pm 8.5)$ \\
\hline 3 & 3 & 52 & $17(32.8 \pm 6.1)$ \\
\hline
\end{tabular}

Table 1. Birth rate of fresh and cold-stored rat sperm. In the IVF, 6 female and 6 male rats were used. Ten vasectomised male rats and 6 pseudo-pregnant rats were used for embryo transfer.

\begin{tabular}{|l|l|l|l|l|l|l|}
\hline Experiment no & No. of inseminated oocytes & No. of fertilised oocytes (\%) & No. of recipients & $\begin{array}{l}\text { No. of transferred fertilised } \\
\text { oocytes }\end{array}$ & $\begin{array}{l}\text { No. of pups (\%) } \\
\text { (\%) }\end{array}$ & $\begin{array}{l}\text { No. GFP positive pups } \\
\text { (\%) }\end{array}$ \\
\hline 1 & 112 & $47(41.2 \pm 21.9)$ & 1 & 24 & $17(70.8)$ & $6(35.3)$ \\
\hline 2 & 160 & $120(44.8 \pm 25.3)$ & 1 & 20 & $13(65.0)$ & $6(46.2)$ \\
\hline Total & 272 & 2 & 44 & $30(68.1)$ & $12(40.0)$ \\
\hline
\end{tabular}

Table 2. Fertilisation rate and birth rate of cold-transported rat sperm. In the IVF, 6 female and 2 male rats were used. Ten vasectomised male rats and 2 pseudo-pregnant rats were used for embryo transfer. 


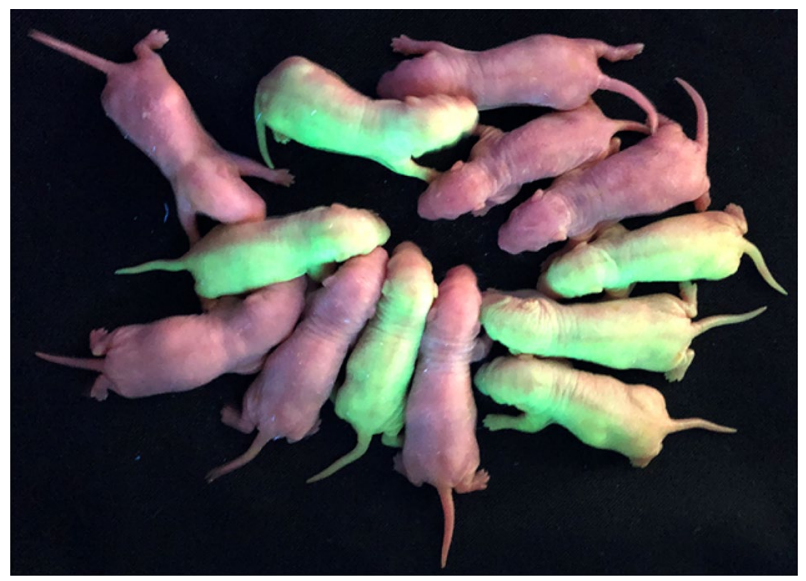

Figure 6. Live pups derived from in vitro fertilisation using cold-transported rat sperm. Fertilised oocytes were transferred into the oviducts of pseudo-pregnant female rats. The embryos developed normally into live pups 22-23 days after embryo transfer. Wild type and GFP-positive pups are shown.

decreased sperm motility ${ }^{12}$. However, adverse effects of DMSO were reported on the motility and integrity of the membranes and acrosome of rat sperm at room temperature ${ }^{13}$. Therefore, further studies are required to identify the optimal solvents as alternatives to DMSO to improve the quality of cold-stored rat sperm.

The total fertilisation rates of rat sperm cold-stored for 1-5 days were lower than that of fresh sperm (84.2\%). On the other hand, the rates of polyspermic fertilisation of cold-stored sperm were lower (1.2-5.2\%) than those of fresh sperm (22.7\%). In a previous study, polyspermic fertilisation was observed in CD (29\%) and Long-Evans $(20 \%)^{14}$ rats. Polyspermy was common in rat IVF using fresh sperm; however, the use of cold-stored rat sperm reduced the rate of polyspermic fertilisation. We assumed the difference in motile activity between fresh and cold-stored rat sperm affected the rate of polyspermic fertilisation. Polyspermy is inhibited by the hardening of the zona pellucida with the surface reaction of the cell membrane of the ooplasm after membrane fusion with sperm and oocyte ${ }^{15}$. Exocytosis of cortical granules and modification of the zona pellucida occur within a few minutes after fertilisation ${ }^{16}$. After exocytosis of the cortical granule, ovastacin and spark inhibited sperm binding to zona pellucida within a few minutes to $30 \mathrm{~min}^{17-22}$. We observed many sperm in the perivitelline space during IVF using fresh sperm, whereas a few sperm existed in the perivitelline space during IVF using cold-stored sperm. Reduced motility and progressive motility of cold-stored sperm may delay the penetration of second or later sperm through the zona pellucida, avoiding polyspermic fertilisation.

Delayed penetration of cumulus cells also reduces polyspermic fertilisation in cold-stored rat sperm. Cumulus cells were dispersed by fresh sperm at $6 \mathrm{~h}$ after insemination, whereas cumulus cells remained with cold-stored sperm. Thus, cold-stored sperm may take longer than fresh sperm to penetrate the layer of cumulus cells. The delayed penetration of cold-stored sperm could be attributed to reduced motility or delayed or dysfunction of the acrosome reaction. Acrosin is an enzyme released from the acrosome during the acrosome reaction ${ }^{23,24}$. Sperm from acrosine-deficient rats passed through the zona pellucida to be fertilised, but had difficulty penetrating the cumulus cells $\mathrm{s}^{2-27}$. Cold-induced acrosine dysfunction may be related to the delayed penetration of cumulus cells in cold-stored sperm. In addition, the delayed penetration of sperm allows sufficient time to react to the modifications of the zona pellucida and positively reduce polyspermic fertilisation in cold-stored sperm. Further studies are required to elucidate the mechanisms involved.

Cold-transport technology provides a practical and efficient method to transport genetically modified rats. In mice, domestic and international transportation have been successfully carried out using the cold-storage technique. Cold transport of rat sperm would be advantageous because rats are difficult to transport as they are more sensitive to environmental changes during transportation and larger than mice ${ }^{28,29}$. Cheaper shipment costs of cold-stored rat sperm would be a beneficial alternative to the shipment of live animals. Some limitations of cold-storage technique about storage condition at refrigerated temperatures and period for 5 days have remained.

Genome editing technology enhances the value of laboratory rats as important models of human diseases and drug development in physiology and toxicology $y^{30}$. As a consequence the production and transport of genetically modified rats will increase over time. We believe the improving the transport system of rat resources using the technology will accelerate multi-institutional collaboration, improve reproducibility of animal science and promote further innovations in biomedical science.

\section{Methods}

Animals. Jcl:SD rats were purchased from CLEA Japan Inc. (Tokyo, Japan) to use as sperm and oocyte donors. Sperm were obtained from male rats (11-15 weeks old) and oocytes were obtained from immature female rats (5-6 weeks old). Crlj:CD(SD) male rats ( $>13$ weeks old) were purchased from Charles River Japan (Kanagawa, Japan) to use as vasectomised male rats for embryo transfer. Crl:CD(SD) female rats (10-12 weeks old) were purchased from Charles River Japan to use as recipients for embryo transfer. Male transgenic rats (SD-Tg(CAG-EGFP)4Osb) and female rats (Slc:SD) purchased from Slc Japan (Shizuoka, Japan) were used for 
cold transport. The breeding environments were light (07:00-19:00 h) and dark (19:00-07:00 h), room temperature was $22^{\circ} \mathrm{C} \pm 2{ }^{\circ} \mathrm{C}$ and with free access to food and water. Male rats were kept in two animals per cage, and female rats were kept in three animals per cage. The Animal Care and Use Committee of Kumamoto University approved the protocols for animal experiments (ID: A2021-025), and all methods were performed in accordance with ARRIVE guidelines and relevant and regulations.

Media and reagents. Lifor was used as cold-storage medium for cauda epididymides (Lifor, Detraxi Inc., boca Raton, Florida, USA). DMSO (FUJIFILM Wako Pure Chemicals Co., Osaka, Japan) was dissolved in Lifor to final concentrations of 5-20\% (v/v). Quercetin (FUJIFILM Wako Pure Chemicals Co., Osaka, Japan) was dissolved in DMSO to final concentrations of 50-300 $\mu \mathrm{g} / \mathrm{mL}$. Modified human tubal fluid (mHTF) was used as sperm collection and IVF medium ${ }^{31,32}$. All media were covered with paraffin oil (Nacalai Tesque Inc., Kyoto, Japan) and aerated with $5 \% \mathrm{CO}_{2}$ at $37^{\circ} \mathrm{C}$ prior to use. Three types of mixed aesthetic agents were prepared by mixing midazolam $(2 \mathrm{mg} / \mathrm{kg})$, medetomidine $(0.375 \mathrm{mg} / \mathrm{kg})$ and butorphanol $(2.5 \mathrm{mg} / \mathrm{kg})$.

Cold storage of cauda epididymides. Cauda epididymides were removed from mature male rats euthanised by cervical dislocation and cauda epididymides were transferred to $1.5-\mathrm{mL}$ plastic tubes containing $1 \mathrm{~mL}$ of cold-storage solution (Lifor + DMSO + quercetin). The tubes were placed in a paper box with a digital thermometer and date logger (Thermochron iButton; Maxim Integrated Products, Inc.). The box was placed in a vacuum bottle (JMK-500, Thermos Co., USA) with two cold packs $(60 \mathrm{~mm} \times 180 \mathrm{~mm})$ precooled in a refrigerator at $4{ }^{\circ} \mathrm{C}$. The bottle was placed in a Styrofoam box $\left(205 \times 125 \times 130 \mathrm{~mm}^{3}\right)$ with four cold packs $(140 \mathrm{~mm} \times 250 \mathrm{~mm})$ precooled in a refrigerator at $4{ }^{\circ} \mathrm{C}$. The packed Styrofoam box stored in a refrigerator at $4{ }^{\circ} \mathrm{C}$ for $0-6$ days. The temperature was measured with a thermometer and was found to be $2-5^{\circ} \mathrm{C}$.

Preparation of sperm. After the cold storage, the cauda epididymides were removed from cold storage solution and the excess solution was gently wiped off with filter paper. Cauda epididymides were washed in saline and transferred to paraffin oil of sperm collection dish. In the paraffin oil, cauda epididymides were made a short incision using microdissecting scissors and sperm were transferred into a $400-\mu \mathrm{L}$ drop of mHTF using glass rod $(15 \mathrm{~mm})$. The sperm suspension was incubated in an incubator at $37^{\circ} \mathrm{C}$ with $5 \% \mathrm{CO}_{2}$. Sperm concentration was calculated using a hemocytometer (Erma, Tokyo, Japan) and sperm suspensions were added to a drop of $200 \mu \mathrm{L}$ IVF medium covered with paraffin oil (the final concentration was $500-1500 \mathrm{sperm} / \mu \mathrm{L}$ ). Sperm were incubated at $37^{\circ} \mathrm{C}$ and $5 \% \mathrm{CO}_{2}$ for $2 \mathrm{~h}$ and used for IVF and assessment of sperm motility.

In vitro fertilisation. Immature female rats were injected with 30 IU equine chorionic gonadotropin (PMSG, ASKA Animal Health Co. Ltd, Japan). At 50-55 h after PMSG injection, the rats were injected with 30 IU human chorionic gonadotropin (hCG, ASKA Animal Health Co. Ltd, Japan). At 16-18 h post-administration of hCG, immature females were euthanised by cervical dislocation and their oviducts were collected. The oviducts were transferred in paraffin oil of the IVF dish containing a sperm suspension. Cumulus oocyte complexes were collected from the ampulla of the oviduct into the IVF drop. The oocytes and sperm were incubated at $37^{\circ} \mathrm{C}$ with $5 \% \mathrm{CO}_{2}$ for $6-7 \mathrm{~h}$. The oocytes were then washed three times with $80 \mu \mathrm{L}$ of mHTF drops covered with paraffin oil and cultured at $37^{\circ} \mathrm{C}$ with $5 \% \mathrm{CO}_{2}$. The oocytes were observed under an inverted microscope 23-24 h after insemination and the fertilisation rate was calculated as the number of fertilised oocytes (monospermic oocytes that showed two pronuclei and a sperm tail or a sperm tail in the cytoplasm and polyspermic oocytes with more than three pronuclei and more than two sperm tails or more than two sperm tails in the cytoplasm were determined to be fertilised oocytes) divided by the total number of oocytes (unfertilised oocyte and fertilised oocyte) and multiplied by 100.

Assessment of sperm motility. The motility of fresh and cold-stored sperm was evaluated using a computer-assisted sperm analyser (IVOS Sperm Analyzer, Hamilton-Thorne Research Co. Ltd., USA). Fresh sperm were diluted to a final concentration of 500 sperm $/ \mu \mathrm{L}$ and cultured in $200 \mu \mathrm{L}$ of mHTF drops covered with paraffin oil for $2 \mathrm{~h}$ at $37^{\circ} \mathrm{C}$ and $5 \% \mathrm{CO}_{2}$. Next, $10 \mu \mathrm{L}$ of the sperm suspension was collected and added to the measurement chamber to measure motility and other parameters. Sperm motility was calculated as the ratio of sperm that moved at a speed of $\geq 5 \mu \mathrm{m} / \mathrm{s}$. Progressive motility was calculated as the percentage of total sperm that progressed $\geq 50 \mu \mathrm{m} / \mathrm{s}$ and had a progressiveness ratio of $\geq 50 \%$. VAP was calculated as the average velocity of the motile sperm. Progressive velocity (VSL) was calculated as the average velocity of motile sperm when measured in a straight line from the start point to the end point. Lateral amplitude (ALH) was calculated as the swing of the sperm head as it moved forward. Beat frequency (BCF) was calculated as the frequency of sperm head crossings relative to the sperm migration path. Straightness (STR) was calculated as how close to a straight line the average migration path of sperm was. Between 500 and 1000 sperm were analysed in each experiment.

Embryo transfer. Embryos obtained by IVF (pronuclear fertilised oocytes and two-cell embryos) were transferred into the oviducts of pseudo-pregnant Crl:CD(SD) female rats ${ }^{33}$. Pseudo-pregnant rats were generated by mating female rats that were judged to be in proestrus by observing vaginal smears with vasectomised Crlj:CD(SD) male rats. Anaesthesia was performed using a mix of three types aesthetic agents administered at a dose of $0.5 \mathrm{~mL}$ per $100 \mathrm{~g}$ of body weight of the rats. Six to twelve fertilised oocytes were placed in one oviduct. After embryo transfer, an antagonist (antisedan, $150 \mu \mathrm{g} / \mathrm{mL}$ ) was administered at a dose of $0.5 \mathrm{~mL}$ per $100 \mathrm{~g}$ of body weight of the rats. The number of live pups was counted after 22-23 days. The birth rate was calculated as the number of live pups divided by the number of embryos transferred and multiplied by 100 . 
Cold transport of rat cauda epididymides. Cauda epididymides were collected from euthanised male rats (SD-Tg(CAG-EGFP)4Osb) at 14:00 h. The epididymides were transferred in a tube filled with Lifor containing $15 \% \mathrm{DMSO}$ and $200 \mu \mathrm{g} / \mathrm{mL}$ quercetin and packed in a cold-transport kit using the same method as described above section of cold storage of cauda epididymides. The cold-transport kit was transported from Niigata University to Kumamoto University via cold transportation (temperature, $5.5-7.5^{\circ} \mathrm{C}$ ) by a shipping company. The transport kit arrived at Kumamoto University after $44 \mathrm{~h}$ and the transported epididymides were stored in a refrigerator at $4{ }^{\circ} \mathrm{C}$ prior to use. After $20 \mathrm{~h}$ storage, the sperm were collected at 06:00 h. At 09:30 h, oocytes were collected from euthanised female rats following superovulation treatment as described in the section above and used for IVF. Between 09:00 and 11:00 h, vaginal smears were confirmed and Crl:CD(SD) female rats in early proestrus were mated with vasectomised $\mathrm{Crlj}: \mathrm{CD}(\mathrm{SD})$ male rats. The number of fertilised oocytes was counted at 15:00 h. The next day, plugged female rats were confirmed between 08:00 and 09:00 $\mathrm{h}$ and used as pseudopregnant rats for embryo transfer. Live pups were counted at 22-23 days after embryo transfer.

Statistical analysis. Statistical analysis was performed using Prism version 8 (GaphPad Software) with arcsine transformation of percentage data, followed by analysis of variance and significant difference testing using Tukey or Dunnett test. Statistical significance was set at $5 \%$ or below the $1 \%$ level.

Received: 6 July 2021; Accepted: 2 November 2021

Published online: 22 November 2021

\section{References}

1. Harrison, C. Mice on the move. Lab Anim. 50, 233-235 (2021).

2. Raspa, M. et al. Dry ice is a reliable substrate for the distribution of frozen mouse spermatozoa: A multi-centric study. Theriogenology 96, 49-57 (2017)

3. Hirabayashi M, Kato M, Ito J, Hochi S. Viable rat offspring derived from oocytes intracytoplasmically injected with freeze-dried sperm heads. Zygote 13, 79-85 (2005).

4. Varisli, O., Agca, C. \& Agca, Y. Short-term storage of rat sperm in the presence of various extenders. J. Am. Assoc. Lab. Anim. Sci. 52, 732-737 (2013).

5. Sarı̈̈zkan, S. et al. The effects of different sugars on motility, morphology and DNA damage during the liquid storage of rat epididymal sperm at $4{ }^{\circ} \mathrm{C}$. Cryobiology 65, 93-97 (2012).

6. Varisli, O., Uguz, C., Agca, C. \& Agca, Y. Effect of chilling on the motility and acrosomal integrity of rat sperm in the presence of various extenders. J. Am. Assoc. Lab. Anim. Sci. 48, 499-505 (2009).

7. Takeo, T. et al. Establishment of a transport system for mouse epididymal sperm at refrigerated temperatures. Cryobiology $\mathbf{6 5}$, $163-168$ (2012).

8. Yoshimoto, H., Takeo, T. \& Nakagata, N. Dimethyl sulfoxide and quercetin prolong the survival, motility, and fertility of cold-stored mouse sperm for 10 days. Biol. Reprod. 97, 883-891 (2017).

9. Johinke, D., de Graaf, S. P. \& Bathgate, R. Quercetin reduces the in vitro production of $\mathrm{H}_{2} \mathrm{O}_{2}$ during chilled storage of rabbit spermatozoa. Anim. Reprod. Sci. 151, 208-219 (2014).

10. Ghaniei, A., Eslami, M., Zadeh Hashem, E., Rezapour, R. \& Talebi, A. Quercetin attenuates $\mathrm{H}(2) \mathrm{O}(2)$-induced toxicity of rooster semen during liquid storage at $4^{\circ} \mathrm{C}$. J. Anim. Physiol. Anim. Nutr. (Berl.) 103, 713-722 (2019).

11. Popham, P. L. \& Novacky, A. Use of dimethyl sulfoxide to detect hydroxyl radical during bacteria-induced hypersensitive reaction. Plant. Physiol. 96, 1157-1160 (1991).

12. Aitken, R. J. \& Baker, M. A. Oxidative stress, sperm survival and fertility control. Mol. Cell. Endocrinol. 250, 66-69 (2006).

13. Si, W., Benson, J. D., Men, H. \& Critser, J. K. Osmotic tolerance limits and effects of cryoprotectants on the motility, plasma membrane integrity and acrosomal integrity of rat sperm. Cryobiology 53, 336-348 (2006).

14. Niwa, K. \& Chang, M. C. Various conditions for the fertilization of rat eggs in vitro. Biol. Reprod. 11, 463-469 (1974).

15. Fahrenkamp, E., Algarra, B. \& Jovine, L. Mammalian egg coat modifications and the block to polyspermy. Mol. Reprod. Dev. 87, 326-340 (2020).

16. Familiari, G., Heyn, R., Relucenti, M. \& Sathananthan, H. Structural changes of the zona pellucida during fertilization and embryo development. Front. Biosci. 13, 6730-6751 (2008).

17. Tokuhiro, K. \& Dean, J. Glycan-independent gamete recognition triggers egg zinc sparks and ZP2 cleavage to prevent polyspermy. Dev. Cell. 46, 627-640.e625 (2018).

18. Burkart, A. D., Xiong, B., Baibakov, B., Jiménez-Movilla, M. \& Dean, J. Ovastacin, a cortical granule protease, cleaves ZP2 in the zona pellucida to prevent polyspermy. J. Cell. Biol. 197, 37-44 (2012).

19. Bleil, J. D., Beall, C. F. \& Wassarman, P. M. Mammalian sperm-egg interaction: Fertilization of mouse eggs triggers modification of the major zona pellucida glycoprotein, ZP2. Dev. Biol. 86, 189-197 (1981).

20. Kim, A. M., Vogt, S., O’Halloran, T. V. \& Woodruff, T. K. Zinc availability regulates exit from meiosis in maturing mammalian oocytes. Nat. Chem. Biol. 6, 674-681 (2010).

21. Que, E. L. et al. Zinc sparks induce physiochemical changes in the egg zona pellucida that prevent polyspermy. Integr. Biol. (Camb.) 9, 135-144 (2017).

22. Que, E. L. et al. Quantitative mapping of zinc fluxes in the mammalian egg reveals the origin of fertilization-induced zinc sparks. Nat. Chem. 7, 130-139 (2015).

23. Adham, I. M. et al. Chromosomal assignment of four rat genes coding for the spermatid-specific proteins proacrosin (ACR), transition proteins 1 (TNP1) and 2 (TNP2), and protamine 1 (PRM1). Cytogenet. Cell. Genet. 57, 47-50 (1991).

24. Kremling, H., Flake, A., Adham, I. M., Radtke, J. \& Engel, W. Exon-intron structure and nucleotide sequence of the rat proacrosin gene. DNA Seq. 2, 57-60 (1991).

25. Isotani, A. et al. A delayed sperm penetration of cumulus layers by disruption of acrosin gene in rats $\dagger$. Biol. Reprod. 97, 61-68 (2017).

26. Adham, I. M., Nayernia, K. \& Engel, W. Spermatozoa lacking acrosin protein show delayed fertilization. Mol. Reprod. Dev. 46, 370-376 (1997).

27. Baba, T., Azuma, S., Kashiwabara, S. \& Toyoda, Y. Sperm from mice carrying a targeted mutation of the acrosin gene can penetrate the oocyte zona pellucida and effect fertilization. J. Biol. Chem. 269, 31845-31849 (1994).

28. Swallow, J. et al. Guidance on the transport of laboratory animals. Lab. Anim. 39, 1-39 (2005).

29. Arts, J. W. M. Transportation in Laboratory Rats Effects of a Black Box 35-66 (Utrecht University, 2016).

30. Neff, E. P. Rats on the rise. Lab Anim. (2021). 
31. Quinn, P., Kerin, J. F. \& Warnes, G. M. Improved pregnancy rate in human in vitro fertilization with the use of a medium based on the composition of human tubal fluid. Fertil. Steril. 44, 493-498 (1985).

32. Kito, S. et al. Improved in vitro fertilization and development by use of modified human tubal fluid and applicability of pronucleate embryos for cryopreservation by rapid freezing in inbred mice. Comp. Med. 54, 564-570 (2004).

33. Nakagata, N. Embryo transfer through the wall of the fallopian tube in mice. Jikken Dobutsu 41, 387-388 (1992).

\section{Acknowledgements}

We thank Prof. Toshikuni Sasaoka (Brain Research Institute, Niigata University) for his helpful advice. The present study was supported by a Grant from the A-STEP (Adaptable and Seamless Technology Transfer Program through Target-Driven R\&D) program of the Japan Science and Technology Agency (feasibility study stage, No. 21447630) and a collaborative research program of Kyudo Co. Ltd.

\section{Author contributions}

K.Y., S.N., N.N., and T.T. designed the study, wrote the initial draft of the manuscript and created the figures. K.Y. and S.N. contributed to the acquisition and analysis of the data. S.N., H.Y., N.M. and E.N contributed to interpretation of the data. S.N., N.N. and T.T. contributed to the analysis, interpretation of the data and revised the manuscript. All authors approved the final version of the manuscript and agree to be accountable for all aspects of the work in ensuring that questions related to the accuracy or integrity of any part of the work are appropriately investigated and resolved.

\section{Competing interests}

The authors declare competing financial interests. The present study was partially supported by a Grant of Kyudo Co. Ltd. K. Y. received a Grant from Kyudo Co. Ltd. N. M. is a member of Kyudo Co. Ltd. N. N. is a member of collaboration laboratory between Kyudo Co. Ltd and Kumamoto University. S. N., H. Y., E. N. and T. T. are no financial interests.

\section{Additional information}

Correspondence and requests for materials should be addressed to T.T.

Reprints and permissions information is available at www.nature.com/reprints.

Publisher's note Springer Nature remains neutral with regard to jurisdictional claims in published maps and institutional affiliations.

(c) (i) Open Access This article is licensed under a Creative Commons Attribution 4.0 International License, which permits use, sharing, adaptation, distribution and reproduction in any medium or format, as long as you give appropriate credit to the original author(s) and the source, provide a link to the Creative Commons licence, and indicate if changes were made. The images or other third party material in this article are included in the article's Creative Commons licence, unless indicated otherwise in a credit line to the material. If material is not included in the article's Creative Commons licence and your intended use is not permitted by statutory regulation or exceeds the permitted use, you will need to obtain permission directly from the copyright holder. To view a copy of this licence, visit http://creativecommons.org/licenses/by/4.0/.

(C) The Author(s) 2021 